\title{
Case Report: The Successful Treatment of High Lethal Dose Paraquat Poisoning With Hemoperfusion
}

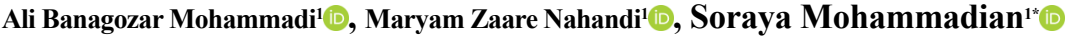

1. Department of Internal Medicine, School of Medicine, Tabriz University of Medical Sciences, Tabriz, Iran.

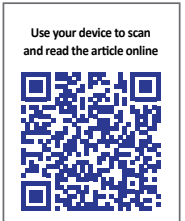

Citation: Banagozar Mohammadi A, Zaare Nahandi M, Mohammadian S. The Successful Treatment of High Lethal Dose Paraquat Poisoning With Hemoperfusion. International Journal of Medical Toxicology and Forensic Medicine. 2020; 10(3):26726. https://doi.org/10.32598/ijmtfm.v10i3.26726

d oi https://doi.org/10.32598/ijmtfm.v10i3.26726

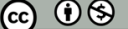

Article info:

Received: 14 August 2019

First Revision: 25 August 2019

Accepted: 01 Jul 2020

Published: 24 Oct 2020

Keywords:

Paraquat, Herbicide, Outcome, Hemoperfusion, Poisoning

\begin{abstract}
Paraquat (PQ), as an herbicide, is mostly used by farmers, especially in the north-west of Iran. Easy access to $\mathrm{PQ}$ is the reason for PQ intoxication in farmers. However, poisoning with $\mathrm{PQ}$ is rare. Most of the PQ-poisoned patients ingest it deliberately and following a suicidal attempt. PQ poisoning treatment has a poor outcome; only a small dose of slightly more than $10 \mathrm{~mL}$ PQ could be harmful and damage lungs forever. Under the conditions of no specific clinical feature, proper history, and diagnostic test, diagnosis is usually difficult. The ingestion of PQ in toxic doses could be fatal, destroying the lungs, kidney, heart, gastrointestinal tract, liver, and other organs. To remove PQ from the blood and intoxicants, one of the best recommendations is Hemoperfusion (HP), as an extracorporeal method. In this case report, according to our treatments, early management of high lethal dose PQ poisoning, especially with HP could reduce the morbidity and mortality rates.
\end{abstract}

\section{Introduction}

he $\mathrm{N}, \mathrm{N}^{\prime}$-dimethyl-4, 4'-bipyridinium

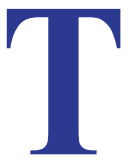
dichloride (paraquat), is a corrosive and highly toxic greenish-black liquid. Despite being banned in numerous countries [1], it remains the second top-selling herbicide in some regions [2]. Its accidental ingestion provides a high mortality rate, and it causes local and systemic toxicity [3]. Here, we reported a case of high-dose Paraquat (PQ) poisoning with a highly positive urine PQ test. The presented patient was successfully recovered with early management using Hemoperfusion (HP) after ingestion.

\section{Case Report}

A 29-year-old healthy male farmer with no medical history or any medication use and drug dependence was presented to Sina Hospital (referral center), 4 hours after an accidental consumption of $90 \mathrm{~mL}$ of PQ $20 \%$ (with the claim that he thought it was Coca-Cola). He vomited immediately after taking it; within 1.5 hours before hospital admission, he experienced 4 vomiting episodes. He was admitted to a local hospital with the chief complaint of several episodes of vomiting. Accordingly, he was given activated charcoal and was managed conservatively with intravenous fluids, antiemetics, and pantoprazole infusion. Then, the patient was referred to our center, promptly.

* Corresponding Author:

Soraya Mohammadian, $M D$.

Address: Department of Internal Medicine, School of Medicine, Tabriz University of Medical Sciences, Tabriz, Iran.

Tel: +98 (41) 35498260

E-mail:smohammadian01@yahoo.com 
At the time of arrival at our center, his main complaints were a sore throat and abdominal pain. On examination, blood pressure was $110 / 70 \mathrm{mmHg}$, heart rate $80 /$ minute, regular respiratory rate of 17 per minute, regular abdominal-thoracic and oxygen saturation of $95 \%$ at room temperature. The temperature was recorded as $98.8^{\circ}$ Fahrenheit by axilla. He was conscious and oriented. Cardiac examination data were normal. Both lung fields were clear on auscultation. Pathologic findings were throat ulcer and severe erythema, LUQ, and epigastric tenderness without rebound guarding. Besides, the sodium dithionite urine test value was equal to +3 .

Endoscopy results suggested erythema in the whole esophagus, the body, and the antrum of the stomach, with erosion in the lower part of the esophagus. Immediately after the patient's admission, 40g N-Acetyl Cysteine daily infusion, 1 gr daily methylprednisolone, 1gr vitamin $\mathrm{C}$ ampule in a single dose, fluid therapy, pantoprazole and metoclopramide ampules, 2 steps of 5-hour hemoperfusion treatment (HA230 hemoperfusion cartridge), and 4-hour hemodialysis by high flux filter were administered. The patient also received 2.5-hour hemoperfusion with 4-hour hemodialysis on the second and third days of hospitalization. Furthermore, $1 \mathrm{gr} /$ day cyclophosphamide was initiated for him for 3 days. The sodium dithionite urine test result was negative after the second day of hemoperfusion.

$\mathrm{N}$-acetyl cysteine infusion and methylprednisolone ampule was discontinued from the 10th day. Moreover, NAC tablets $2400 \mathrm{mg} / \mathrm{d}$ divided into 4 doses, and $50 \mathrm{mg}$ prednisolone tablets daily were replaced in the patient's medication orders. He was discharged with decreasing NAC, methylprednisolone, and AlMg-S syrup after 12 days. Prednisolone and NAC 30 were discontinued 24 and 30 days after the poisoning, respectively.

He was paraclinically evaluated up to 1.5 months after discharge, and the following items checked were as follows: Hb, Hct, WBC, Plt, MCV, MCH, MCHC, Urea, $\mathrm{Cr}, \mathrm{Na}, \mathrm{K}, \mathrm{PT}$, PTT, AST, ALT, ALP, BS, PH, PCO2, $\mathrm{HCO} 3, \mathrm{PO} 2, \mathrm{O} 2 \mathrm{Sat}$, qualitative sodium dithionite test (urine paraquat test); however, during this period, the only abnormal findings were as follows. Creatinine was equal to 0.9 on the first day; however, it increased from the second day and reached 3.4 on the seventh day. Then, it dropped over 10 days and became normal. Serum urea started increasing since the fourth day of poisoning and it decreased from the tenth day. The urine PQ test was highly positive on the first day and slightly positive on the second day. Additionally, Po2 dropped from 82 to 46 on the second day, but rose again on the third day to 73 ; in other days, it reached over 84 . The patient received regular follow-up sessions until 25 months after poisoning and during this period he had no complication. Considering his favorable general condition, he was reluctant to perform paraclinical procedures. There was no pathologic finding on examination.

\section{Discussion}

PQ is commercially sold in the form of liquid concentrate with a concentration range of $20 \%$ to $42 \% \mathrm{w} / \mathrm{w}$ [4] Furthermore, products containing PQ in combination with other herbicides, like sodium chlorate and 2,4-dimethylamine are available in the market [4]. The issue is that PQ causes critical injury in the kidney while it is eliminated by this organ. Despite treatment, the ingestion of a small dose of PQ could severely damage the lung and kidney [5]. Being a bipyridyl compound, PQ directly damages cells by the production of superoxide radicals or other reactive oxygen species and nitrite radicals [6] The oral ingestion of $<20 \mathrm{mg} / \mathrm{kg}$ of PQ $(7.5 \mathrm{cc} 20 \%$ ) may result in the erosions of the tongue, oral mucosa, and fatal damage to the GI tract. With the consumption of 20-50 $\mathrm{mg} / \mathrm{kg}$ of PQ (7.5-18.5cc 20\%), renal tubular necrosis, hepatic necrosis, and pulmonary fibrosis may occur with moderate toxicity; it usually ends in death in 2-3 weeks.

Consuming $>50 \mathrm{mg} / \mathrm{kg}$ of PQ (18.5cc $20 \%$ ), usually leads to death in 1-3 days, following multi-organ dysfunction and shock [7]. Early diagnosis with prompt history taking and aggressive treatment of PQ poisoning with HP could eliminate PQ effects and reduce mortality [5]. The elimination of PQ is almost conducted by kidneys, usually within $24 \mathrm{~h}$ in minor poisonings. However, the terminal elimination half-life could exceed $100 \mathrm{~h}$ [8]. The referred patient presented 4 hours after the consumption of the poison; therefore, the urine PQ levels could be tested and the diagnosis was based on history, clinical, and laboratory findings, as well as the documentation of the consumption of the poison.

A specific antidote of PQ does not exist and a small amount of this poison results in a fatal outcome. The management of this poisoning agent is supportive and gastric lavage; adsorbents, such as activated charcoal (1-2 $\mathrm{g} / \mathrm{kg})$ and Fuller's earth $(1-2 \mathrm{~g} / \mathrm{kg})$ should be initiated as early as possible to prevent the absorption of the poison [9]. Antioxidants, such as vitamins $\mathrm{C}$ and $\mathrm{E}$ and $\mathrm{N}$-acetyl cysteine, a free radical scavenger, have also been used in this respect. Hemoperfusion has shown to be effective in decreasing the PQ level if given within 4-6 h of ingestion. Hemodialysis is only used as a supportive treatment for patients who develop acute tubular necrosis. 
The role of immunosuppression is controversial and under investigation. Some meta-analysis concluded that patients who received glucocorticoid with cyclophosphamide in addition to standard care may generate a lower risk of death at final follow-up, compared to those receiving standard care alone; however, other research failed to support that claim $[10,11]$.

The therapeutic outcome depends on the severity of the poisoning and the time taken to avail of medical procedures. The high mortality rates are due to the toxicity of the compound and the lack of a specific antidote. Young age, percutaneous or inhalational route, negative PQ test, exposure to less PQ, and fewer degrees of leukocytosis, acidosis, renal, hepatic, and pancreatic failures on admission are good prognostic factors. PQ ingestion may provide late complications, such as oropharyngeal ulceration, esophageal erosions, esophagitis, renal failure, pulmonary fibrosis, and strictures [12, 13].

Treating such cases remains supportive; therefore, leading to high mortality. This poison always results in respiratory and renal damage after the consumption of an unexplained dose. The survival of our patient could be explained by several episodes of vomiting; the lack of any lung, pancreatic, or hepatic failure, acidosis, or other severe End Organ damage due to the early management of preventing the poison absorption, and prompt initiation of favorable supportive treatment. The most important of all, the rapid start of hemoperfusion with hemodialysis could be highly effective $[6,12]$.

\section{Conclusion}

Early diagnosis with prompt history taking and aggressive management of $\mathrm{PQ}$ poisoning with hemoperfusion could reduce the associated mortality rate. A small amount of PQ poison leads to a fatal outcome. There is no specific antidote to this poison. The late complications of $P Q$ ingestion are severe and disabling. Thus, the healthcare team should always think of this poison in case of respiratory and renal damage after unexplained poisoning history. Further studies are required on the effects of HA230 hemoperfusion cartridge in PQ poisoning.

\section{Ethical Considerations}

\section{Compliance with ethical guidelines}

All ethical principles were considered in this article. This case report was in accordance with the ethical standards of the institutional and/or national research committee and with the 1964 Helsinki declaration and its later amendments.

\section{Funding}

This research did not receive any grant from funding agencies in the public, commercial, or non-profit sectors.

\section{Author's contributions}

All authors contributed in preparing this article.

\section{Conflict of interest}

The authors declared no conflict of interest.

\section{Acknowledgements}

We would like to thank the Clinical Development Research Unit of Sina Educational, Research and Treatment Center, Tabriz University of Medical Sciences, Tabriz, Iran; for their assistance in this research.

\section{References}

[1] Zaare Nahandi M, Banagozar Mohammadi A. [Ban the sale of paraquat: General health promotion (Persian)]. Hakim Health Sys Res. 2016; 19(2):69-71. http:/ /hakim.hbi.ir/article-1-1677-fa.html

[2] Saravu K, Sekhar S, Pai A, Barkur AS, Rajesh V, Earla JR. Paraquat - A deadly poison: Report of a case and review. Indian J Crit Care Med. 2013; 17(3):182-4. [DOI:10.4103/09725229.117074] [PMID] [PMCID]

[3] Raghu K, Mahesh V, Sasidhar P, Reddy PR, Venkataramaniah V, Agrawal A. Paraquat poisoning: A case report and review of literature. J Family Community Med. 2013; 20(3):198200. [DOI:10.4103/2230-8229.122023] [PMID] [PMCID]

[4] Jagadeesan M, Nithyananthan P, Banupriya M, Mahendrakumar K, Prasanna Karthik S, Kannan R. A study on clinical profile of paraquat poisoning in a tertiary care hospital. Int J Adv Med. 2017; 4(4):1088-91. [DOI:10.18203/2349-3933.ijam20173237]

[5] Li H, Song L, Jian X. Efficacy of hemoperfusion therapy in patients with paraquat poisoning: A meta-analysis. Int J Clin Exp Med. 2017; 10(8):11371-81. http://www.ijcem.com/ files/ijcem0053106.pdf

[6] Ostadi A, Paeezi M. Hemoperfusion efficiency in paraquat toxicity. Int J Med Toxicol Forensic Med. 2018; 8(4):151-6 [DOI:10.22037/ijmtfm.v8i4(Autumn).23089]

[7] Shi Y, Bai Y, Zou Y, Cai B, Liu F, Fu P, Wang L. The value of plasma paraquat concentration in predicting therapeutic effects of haemoperfusion in patients with acute paraquat poisoning. PLoS One. 2012; 7(7):e40911 [DOI:10.1371/journal. pone.0040911] [PMID] [PMCID]

[8] Wunnapuk K, Mohammed F, Gawarammana I, Liu X, Verbeeck RK, Buckley NA, et al. Prediction of paraquat exposure and toxicity in clinically ill poisoned patients: A model 
based approach. Br J Clin Pharmacol. 2014; 78(4):855-66. [DOI:10.1111/bcp.12389] [PMID] [PMCID]

[9] Suntres ZE. Role of antioxidants in paraquat toxicity. Toxicology. 2002; 180(1):65-77. [DOI:10.1016/S0300-483X(02)00382-7]

[10] Li LR, Sydenham E, Chaudhary B, Beecher D, You C. Glucocorticoid with cyclophosphamide for paraquat-induced lung fibrosis. Cochrane Database Syst Rev. 2014; (6):CD008084. [DOI:10.1002/14651858.CD008084.pub4] [PMID]

[11] Gawarammana I, Buckley NA, Mohamed F, Naser K, Jeganathan K, Ariyananada PL, et al. High-dose immunosuppression to prevent death after paraquat self-poisoning-a randomised controlled trial. Clin Toxicol (Phila). 2018; 56(7):6339. [DOI:10.1080/15563650.2017.1394465] [PMID]

[12] Li A, Li W, Hao F, Wang H. Early stage blood purification for paraquat poisoning: A multicenter retrospective study. Blood Purif. 2016; 42(2):93-9. [DOI:10.1159/000445991] [PMID]

[13] Liu XW, Ma T, Li LL, Qu B, Liu Z. Predictive values of urine paraquat concentration, dose of poison, arterial blood lactate and APACHE II score in the prognosis of patients with acute paraquat poisoning. Exp Ther Med. 2017; 14(1):79-86. [DOI:10.3892/etm.2017.4463] [PMID] [PMCID] 